\title{
User Association Strategy for Energy Efficiency and Interference Mitigation of Heterogeneous Networks
}

\author{
Samira Achki $\mathbb{D}^{1}{ }^{1}$ Layla Aziz $\mathbb{D}^{1},{ }^{1}$ Fatima Gharnati, ${ }^{2}$ and Abdellah Ait Ouahman ${ }^{2}$ \\ ${ }^{1}$ Computer Science Department, Cadi Ayyad University-FSTG, Marrakesh, Morocco \\ ${ }^{2}$ National School of Applied Sciences, Marrakech, Morocco \\ Correspondence should be addressed to Samira Achki; s.ichki@gmail.com and Layla Aziz; layla.az1@gmail.com
}

Received 8 February 2020; Accepted 9 April 2020; Published 22 May 2020

Academic Editor: Michelina Catauro

Copyright ( 2020 Samira Achki et al. This is an open access article distributed under the Creative Commons Attribution License, which permits unrestricted use, distribution, and reproduction in any medium, provided the original work is properly cited.

The necessity of the use of several mobile services increases every day. This requires the consumption of energy rates. Hence, minimizing the energy and interferences of mobile users are major concerns of Heterogeneous Networks (HetNet). This paper aims to improve the energy of mobile users by minimizing a weighted function. To consider significant weights values, we have exploited the multicriteria AHP method. The cost function represents the cost of users toward available BS. It includes the essential criteria influencing energy consumption. Moreover, we introduce an optimal user association based on their minimal cost values. Simulation results prove that the optimal method has saved more energy and reduced network interferences.

\section{Introduction}

HetNets are considered to offer added capacity and more spectral efficiency. HetNet is a grouping the different cell kinds: Macro, Picos and femtoscell, and divided access technology. Picocells and femtocells named small cells are densely populated and distributed in the geographic area of the macro cell. These small cells [1] use less power as they present end users are nearer to network access points. That way, one of the key objects for HetNet is to improve energyefficient and reduce the mitigation interference of small cells. The mobile wireless communication, which necessitates high energy efficiency (EE), great system capacity, less interference and strong signal coverage, and, have contracted rapidly and experienced explosive data evolution over the last period, [2]. Because by this trend, satisfying the improved EE, mitigation interference and improved quality of service (QoS), the HetNet nowadays has to transport divers data information to the users with higher energy-efficiency. So, the architecture of the multi-tier heterogeneous network (HetNet), based on small cells, has been propounded as a promising technology to grow and increase the network capacity and avert coverage holes [3]. Also, due to the swiftly increase energy consumption in wireless systems, the research study of EE has concerned broad attention all over the world $[4,5]$. It is known that HetNet with small cells can resolve the physical poverty of radiofrequency and growth the system capacity to some level, but at the purse of the extent and deploying more hardware infrastructures, that mains to higher energy consumption accordingly [6]. Therefore, owing to the compromise between the profit and cost, the EE and management of the intereference of HetNet is enough interesting and dignified of research, that is primarily discussed in this paper. In HetNet, the greater density deployed user mobile and small cells are frequently lightly charged because of their base transmit power associated with macro base stations (BSs). Orderly to take full benefit and supervised the signal transmission between user's mobile MUEs and macro, smallcells. In this paper, we explore a new algorithm based on a cost function for user association in HetNet. We have exploited the AHP method for attributing a significant values of weights in the cost function. We have taken into account three important factors: SINR, distance, and the power consumed by the user. Motivated by the importance of enhancing energy efficiency and making an optimal association of users, we have formulated our problem as a minimization problem. Moreover, we have exploited the AHP process for attributing the weights' preferences in the proposed cost function, where three 


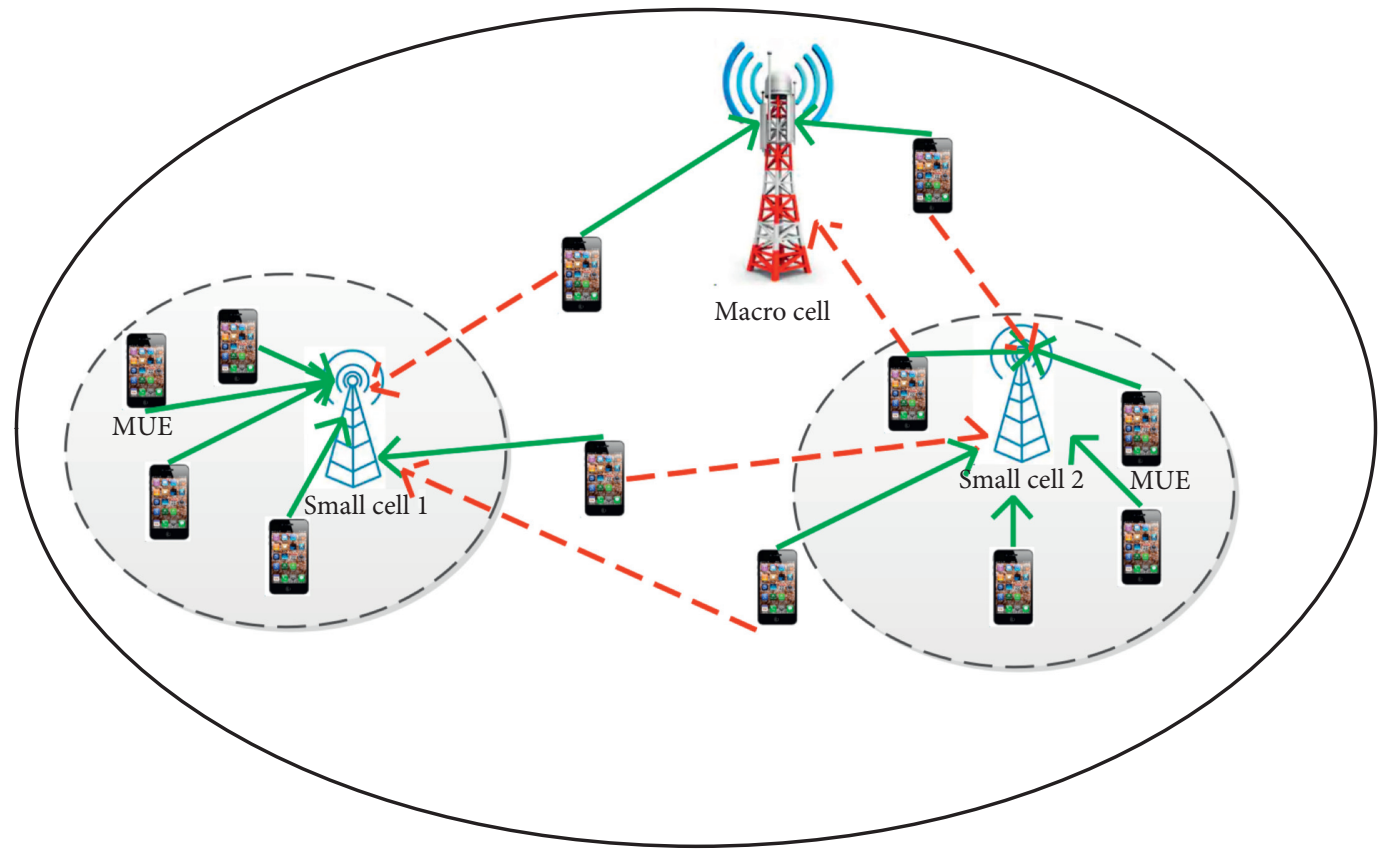

FIgURE 1: The system model for uplink transmission in HetNet.

TABLe 1: Criteria importance meaning.

\begin{tabular}{lc}
\hline Relative importanc & Meaning \\
\hline 1 & Equal \\
3 & Weak \\
5 & Strong \\
7 & Demonstrated over the others \\
9 & Absolute \\
\hline
\end{tabular}

essential criteria are considered. This paper is organized as follows: Section 2 reviews the most popular and recent approaches used for improving the energy efficiency in HetNets. Section 3 presents the main contributions of this work. Section 4 details the problem formulation and the numerical illustration. Section 5 presents the results of the studied scenarios. Section 6 concludes the present study.

\section{Related Works}

In recent years, the research including improved EE and mitigation of interference based on SINR in HetNet has concerned more interests all to the world. On EE, significant results previously exist to save battery power as well as small and macro Base Station (BS) transmission power. In [7], the authors proposed an algorithm to resolve the nonlinear mixed-integer programming problem where is combined cell activation and selection scheme for energy efficiency and developed a three-layer iterative. In [8] exploited the weighted sum of rates in the long-term to stability the network weight and therefore realized developed load balancing benefit and energy efficiency. In [9] explored schemebased BS association for heterogeneous networks and proposed a distributed charge update approach based on algorithm a coordinate descent. In [10] suggest a quality of service (QoS) determined distributed cell association
TABLE 2: RCI values.

\begin{tabular}{lc}
\hline Criteria number & RCI values \\
\hline 1 & 0 \\
2 & 0 \\
3 & 0.58 \\
4 & 0.90 \\
5 & 1.12 \\
6 & 1.24 \\
7 & 1.32 \\
8 & 1.41 \\
9 & 1.45 \\
10 & 1.49 \\
\hline
\end{tabular}

algorithm in that users obtain a number of resource blocks which is only adequate to satisfy their QoS constraints while exploiting the sum purpose of rate. In [11] proposed algorithm that combined user scheduling and user association for load balancing and EE by maximizing a distributed technique the optimization named the alternating direction method of multipliers. In [12] proposed algorithm the utility that based on user association which practices an almost technical based on subframe interference coordination. In [13] proposed algorithm a distributed certainty propagation to determine user association problems in HetNet with several user significances. In [14] proposed many schemes of user association based on weighted average rate using stochastic geometry approach and on cover probability. In [15] proposed algorithm of power control with mobility prediction to evaluate far radio head switching processes in a heterogeneous cloud radio access network (H-CRAN) to enhance energy efficiency. In [16] presented a joint optimization approach that considers the BS sleep model for ameliorating the energy-efficiency of HetNet. In [17] elaborate algorithm a Small-BS on/off switching based on the 


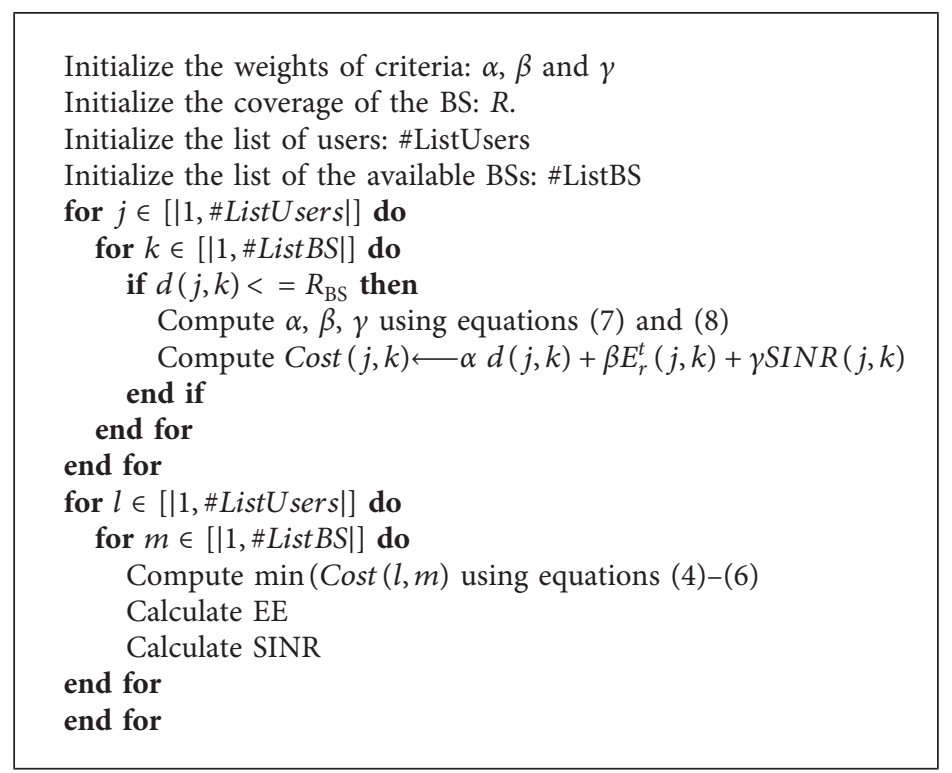

Algorithm 1: Optimal user association.

TABLE 3: Matrix of criteria importance.

\begin{tabular}{lccc}
\hline Criteria & C1 & C2 & C3 \\
\hline C1 & 1 & 7 & 9 \\
C2 & 0.14 & 1 & 3 \\
C3 & 0.11 & 0.33 & 1 \\
\hline
\end{tabular}

TABle 4: Normlized matrix.

\begin{tabular}{lccc}
\hline Criteria & $\mathrm{C} 1$ & $\mathrm{C} 2$ & $\mathrm{C} 3$ \\
\hline C1 & 0.80 & 0.84 & 0.69 \\
C2 & 0.112 & 0.120 & 0.23 \\
C3 & 0.08 & 0.039 & 0.07 \\
\hline
\end{tabular}

optimum distance to decrease power consumption in the network. In [18] considered a small-cell sleep methodology based on the traffic load in that BSs are put off for a certain duration when their traffic load reductions under some threshold. In [19] proposed an energy-efficient growth problem based on coverage probability below also a random sleeping strategic sleeping policy and purpose static and dynamic user models in the sleeping policy strategic, that only take on considered BS on/off strategies but not take the user association. In [20] proposed a Markov theory for Mobility Model based on Velocity Organization (MM-VC) and studies the characteristics of the phase-type distribution of cell resident time. Also, the sleeping strategy between small cells was useful to decrease the energy consumption of the system. However, it only measured energy consumption and canceled the throughput of the network. Moreover, it did not consider the user association. In [21] proposed strategies that corporate optimization method considering User Association and Small-Cell Base Station On/Off Strategies for Energy Efficiency. The authors in [22] optimized the Resource Allocation (RA) problem in the Internet of things (IoT) based on the heuristic algorithm, this algorithm aims to
TABLE 5: Weighted matrix.

\begin{tabular}{lccc}
\hline Criteria & C1 & C2 & C3 \\
\hline C1 & 0.77 & 1.07 & 0.61 \\
C2 & 0.10 & 0.15 & 0.20 \\
C3 & 0.08 & 0.05 & 0.06 \\
\hline
\end{tabular}

TABLE 6: Simulation parameters.

\begin{tabular}{lc}
\hline Parameter & Value \\
\hline Number of macro BS & 1 \\
Number of small BS & 2 \\
Users number & $30-60-90$ \\
Noise power & $-174 \mathrm{dBmHz}$ \\
Power of transmission (macro BS) & $46 \mathrm{dBm}$ \\
Power of transmission (small cell 1) & 20 \\
Power of transmission (small cell 2) & 25 \\
Pathloss & $128,1+37 \log _{10}(\mathrm{~d})$ \\
\hline
\end{tabular}

achieve the goal of optimizing the RA and decreasing the total communication cost between resources and gateways. In [23], the authors proposed the Bat Algorithm (BA) for reducing the energy consumption in a wireless sensor network. This algorithm aims to select the best monitoring sensor node in the path to decreasing energy consumption. Moreover, its main objective is to cover the network lifetime through covering the lifetime of operating sensors as well as transferring collected data from great node to the sink. The authors in [24] proposed the method of machine learning paradigm applying PositionBased Services (PBSs) confidentiality via the mobile edge computing in the real-time industrial informatics, to assure the timely delivery of position-based services at the network edge. The profits of mobile edge service policy proposal position privacy and poor latency by a resource 


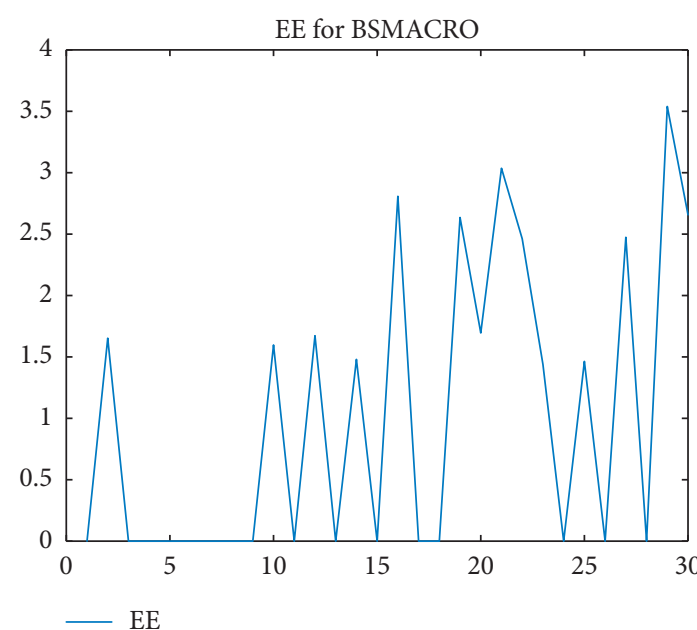

(a)

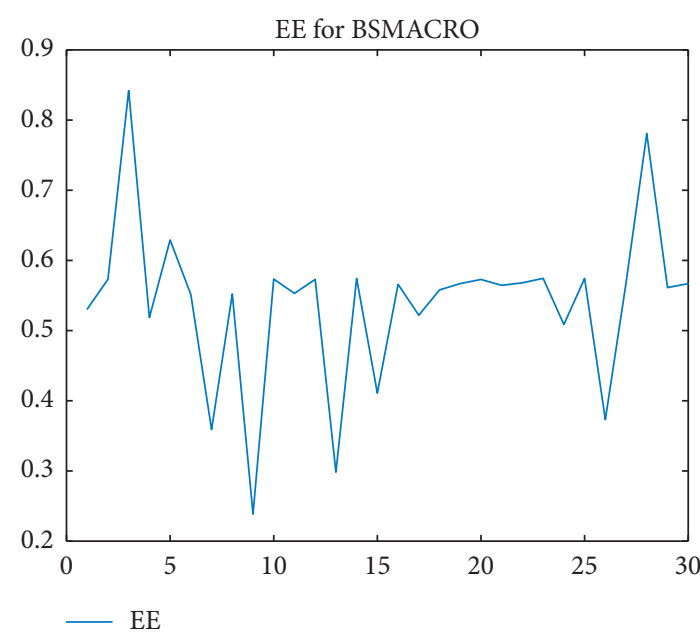

(b)

Figure 2: The energy efficiency (a) and SINR (b) of macro BS for 30 MUE.

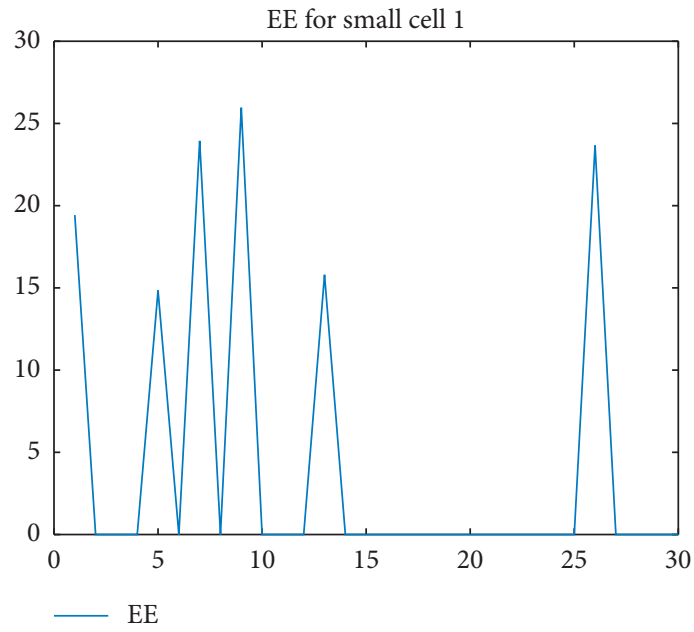

(a)

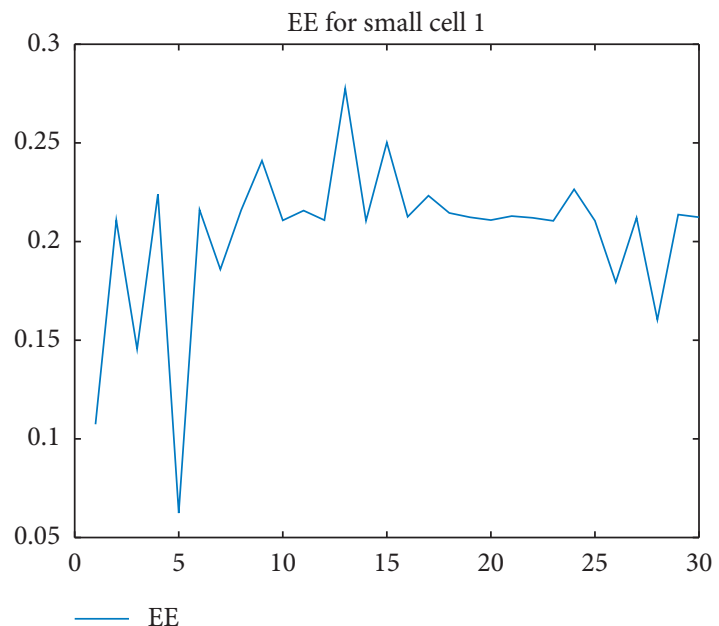

(b)

Figure 3: The energy efficiency (a) and SINR (b) of small BS 1 for 30 MUE.

of networking and computing services at the locality of roaming users. In [25], authors proposed the energyaware green model for Cyber-Physical Security (CPS) in a smart industrial system through achieving confidentiality, the main goal is to improve its energy consumption. So the proposed model that runs on real-time taking into account the regressive position-based query scheduling to decrease the communication and computation cost for each query. Hence, this eases the energy consumption optimization. Several studies have been focused on the integrated multi-criteria tools, where different multicriteria methods have been combined for achieving a significant selection. Among these combined tools AHPTOPSIS and Fuzzy-AHP tools are widely exploited. To maximize the network lifetime in WSN, authors in [26] proposed an integrated AHP-TOPSIS tool. The combined model aims to select the clusters' leaders efficiently.

\section{Authors' Contributions}

In this section, The authors present the main contributions of this work:

(i) The above studies generally take attention to the combined optimization algorithms between BSs strategies and user association. Though, all of the aforementioned analyses did not focus necessarily on joint optimization algorithms of user association and Interference strategy.

(ii) The majority of the previously achieved works determine the criteria importance using equal or random preferences of criteria. However, the present study focuses on a novel selection algorithm based on the AHP method to determine the significant importance of the essential criteria. 


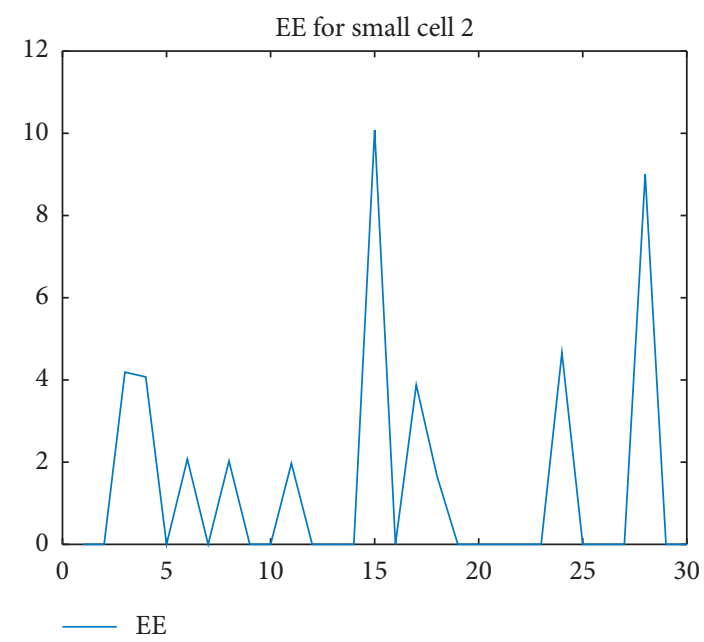

(a)

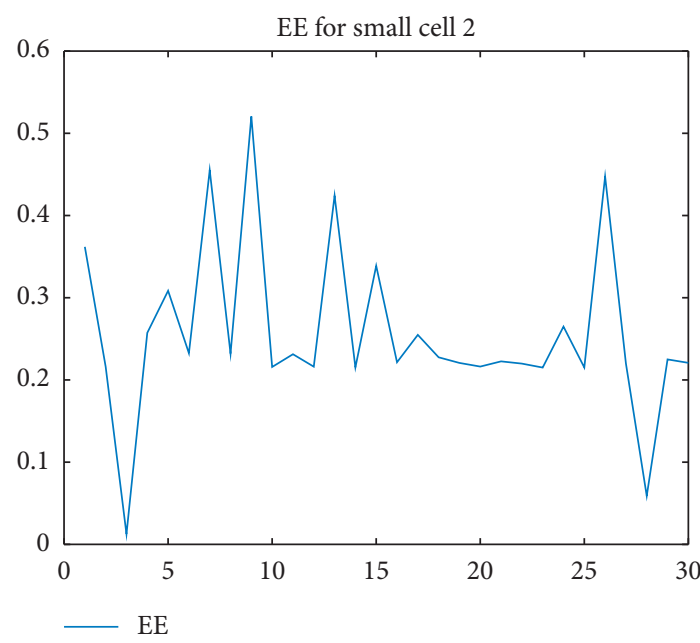

(b)

FIgUre 4: The energy efficiency (a) and SINR (b) of small BS 2 for 30 MUE macro.

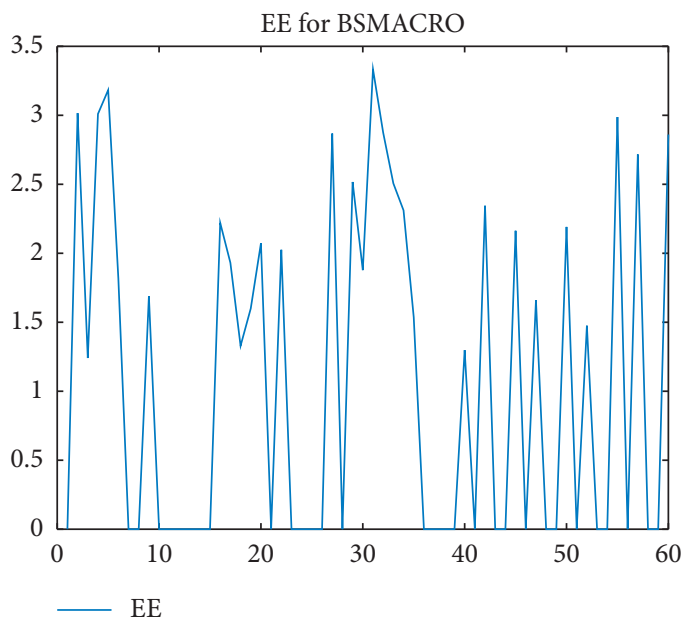

(a)

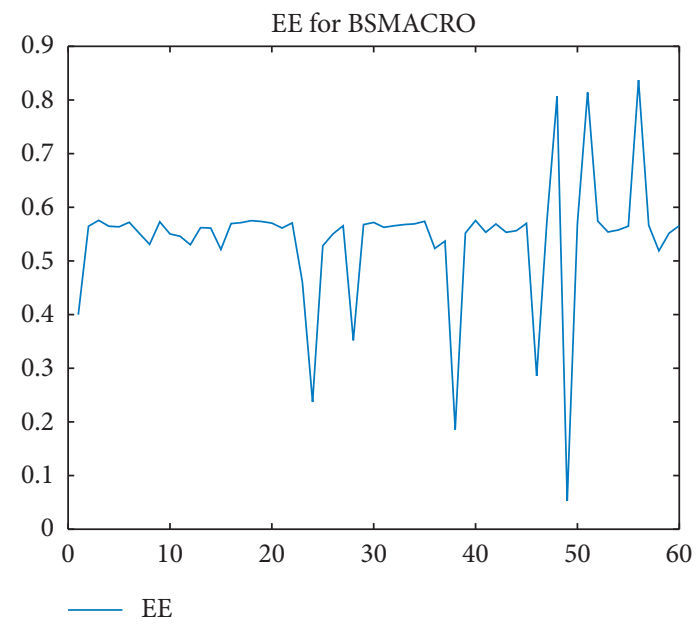

(b)

FIGURE 5: The energy efficiency (a) and SINR (b) of macro BS for 60 MUE.

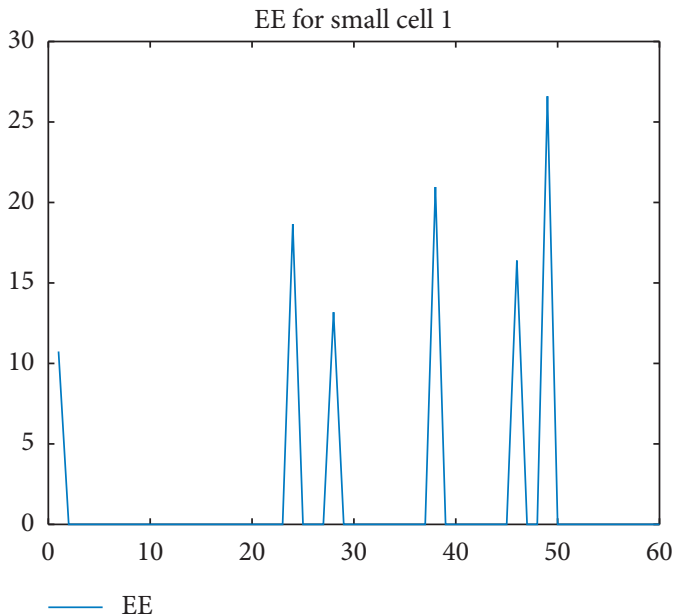

(a)

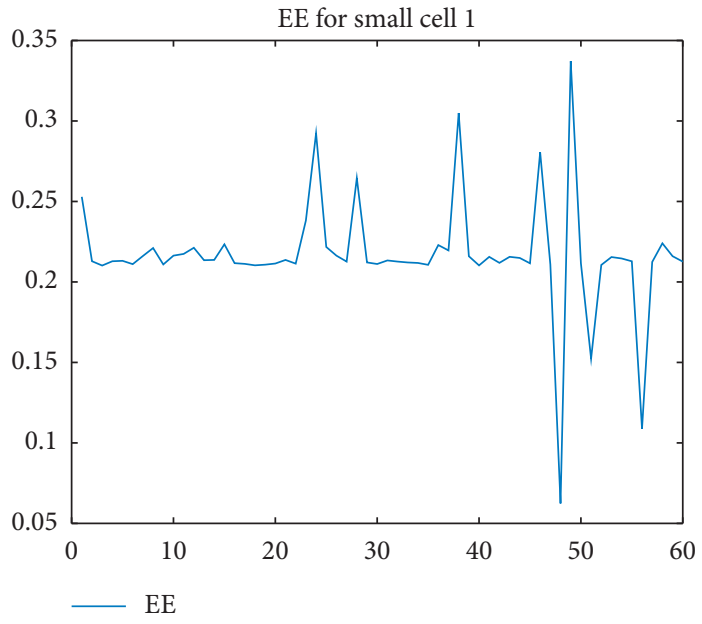

(b)

FIgURE 6: The energy efficiency (a) and SINR (b) of small BS 1 for 60 MUE. 


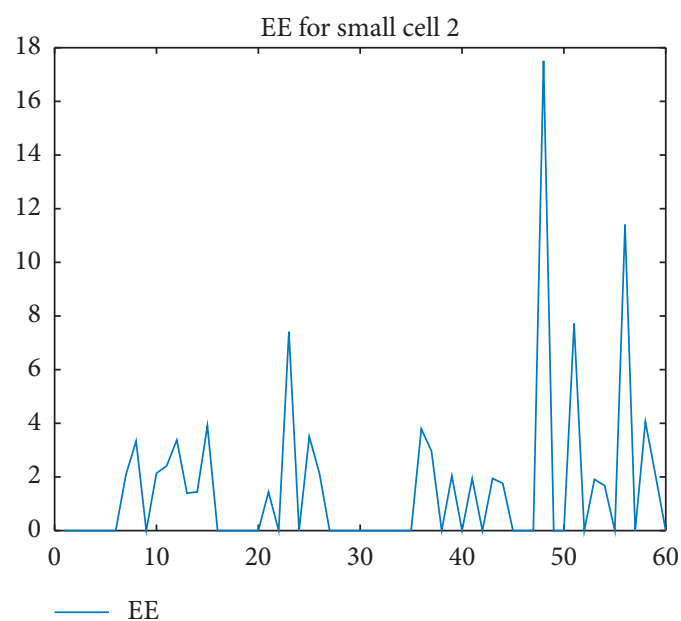

(a)

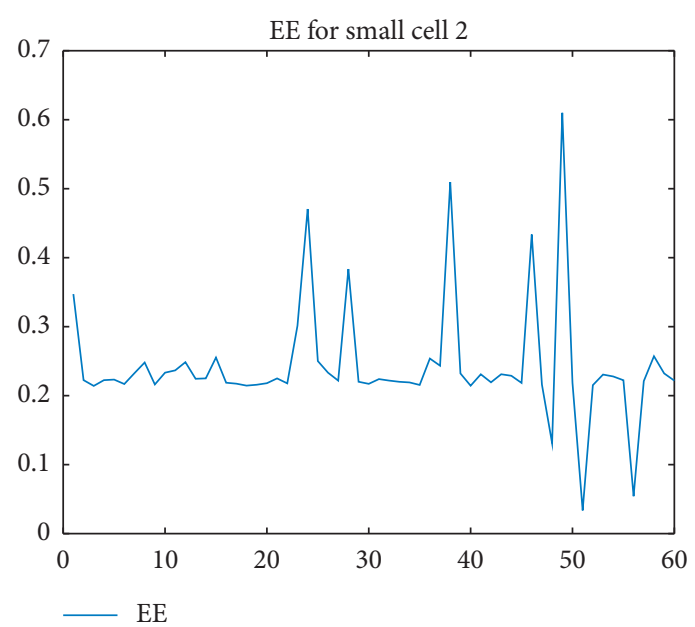

(b)

Figure 7: The energy efficiency (a) and SINR (b) of small BS 2 for 60 MUE.

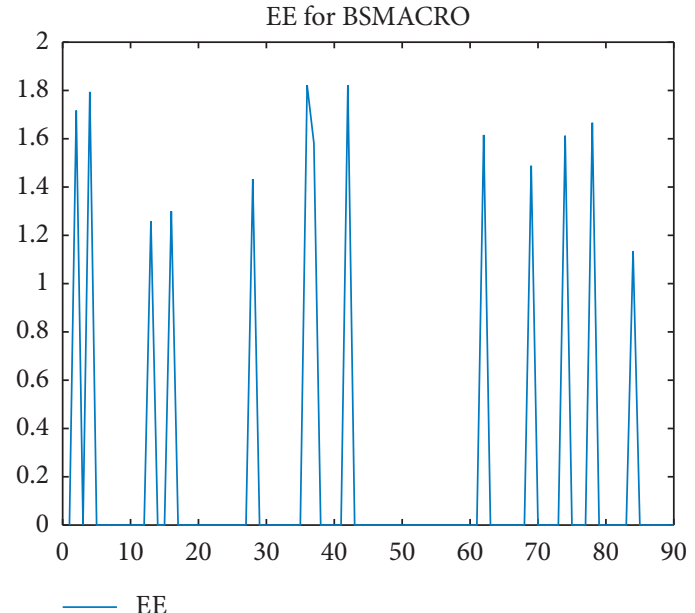

(a)

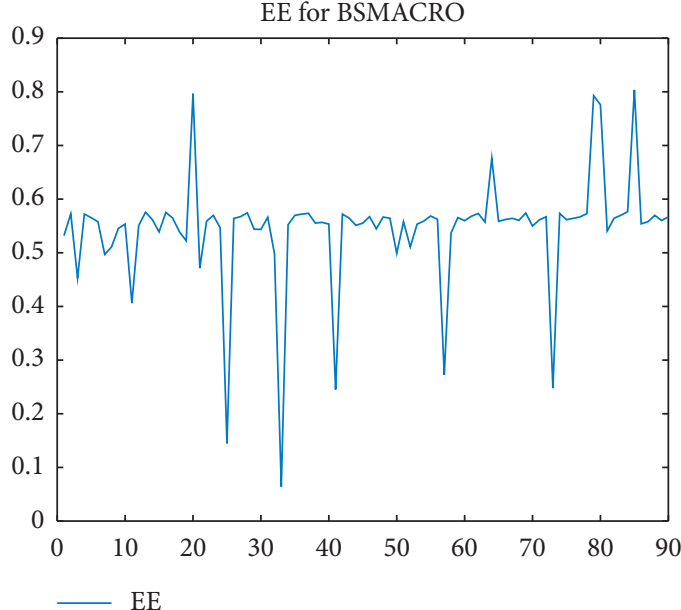

(b)

Figure 8: The energy efficiency (a) and SINR (b) of macro BS for 90 MUE.

(iii) From our knowledge, this work is the first one that combines the multi-criteria AHP method and an optimization algorithm for minimizing the cost of the users association and resolving the problem of network interferences.

\section{Proposed Work}

4.1. System Model and Problem Formulation. The system model illustrates in Figure 1 contains one Macro base station (Macro Cell) underlaid with two Small base station (Small Cell). The user mobile (MUEs) is randomly positioned in the coverage area of the Macro Cell, while the MUEs are randomly located in their associated Small Cells.

In our model, Users are characterized by various criteria where each one influences the user association. The main objective of this work is enhancing energy efficiency by reducing the wireless interferences and making an efficient user association considering several criteria. the SINR of MUE $i$, associated with its designated MBS $k$ can be written as [27]:

$$
Y_{i}^{k, m}=\frac{P_{m}^{i} G_{i}^{k, m}}{\sum_{j \in I_{m}} P_{j}^{m} G_{j}^{m}+\sum_{r \in I_{f}} P_{r}^{f} G_{r}^{f}+\sigma^{2}},
$$

the EE of the $n$th UE which selects the mth BS can be written as [28]:

$$
\mathrm{EE}_{m, n}=\frac{B \log _{2}\left(1+Y_{m, n}\right)}{P_{n}^{\text {sum }}},
$$

where: $\varepsilon$ represents the power coefficient and $\varepsilon \in[0 ; 1]$, $P_{n}^{\text {sum }}=P_{\mathrm{CU}}+\varepsilon P_{n}^{T}$

In order to include the different criteria for making users association, we consider the following weighted function:

$$
f(u)=\alpha * d+\beta * E+\gamma^{*} \operatorname{SINR},
$$




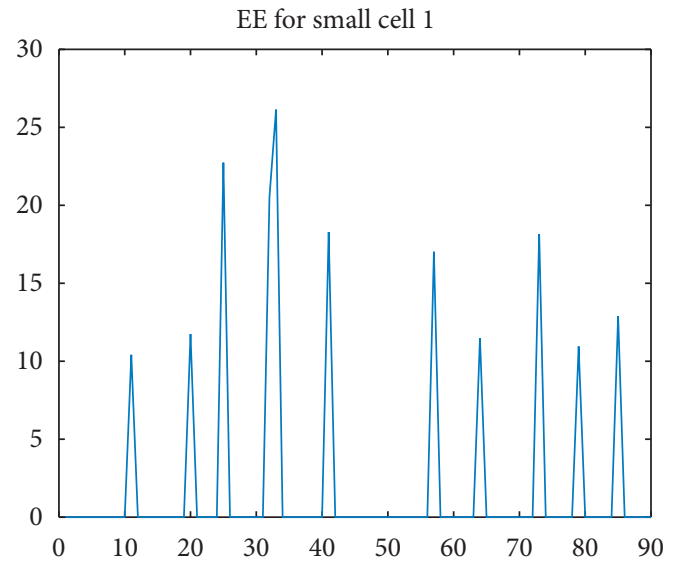

(a)

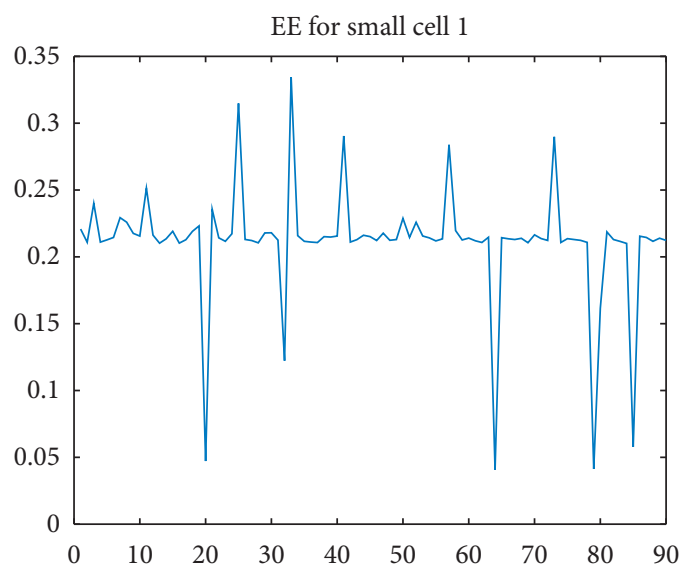

(b)

FIGURE 9: The energy efficiency (a) and SINR (b) of small BS 1 for 90 MUE.

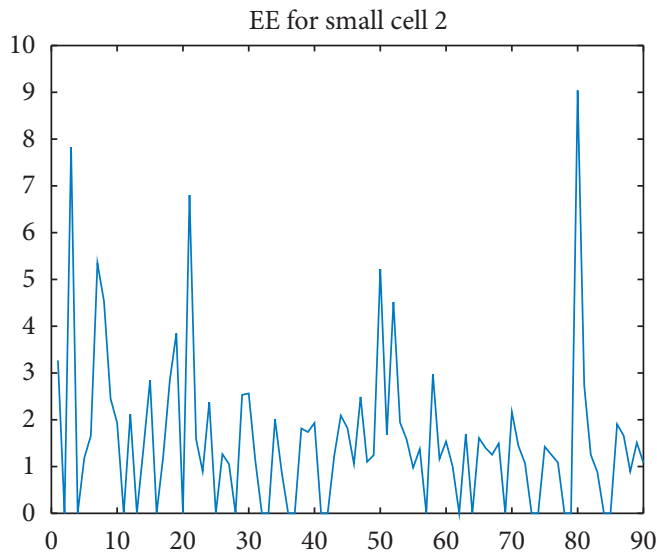

(a)

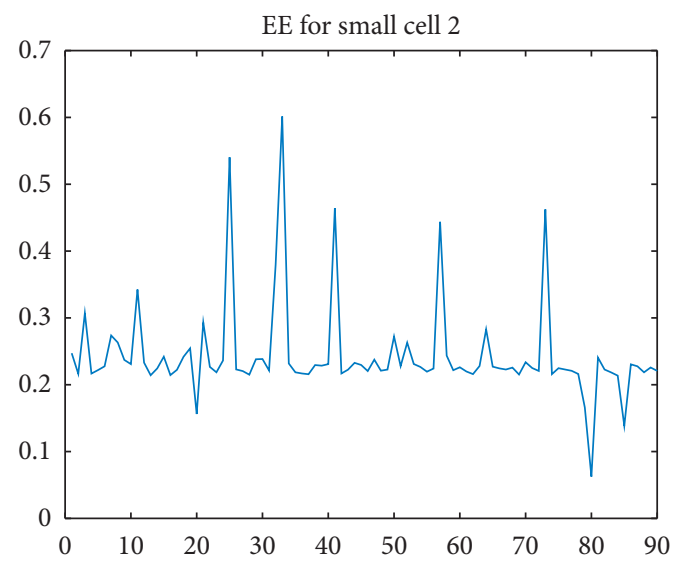

(b)

FIGURE 10: The energy efficiency (a) and SINR (b) of small BS 2 for 90 MUE.

where $\alpha$ represents the weight associated with energy criteria $(E) . \beta$ is the weight of the distance separating the user from the BS. $\gamma$ is the weight of the SINR criteria.

Each user has three essential criteria (distance from BS, its power and the value of its SINR) where each one impacts its value. In this work, we assume that each user disposes of an association cost. So, the association of all users composes a cost matrix. The main objective of the user association problem is designing an optimal association technique. Therefore, our problem is minimizing the cost of the users association. We express our problem as follows:

$$
\min f(u)
$$

subject to:

$$
\begin{aligned}
& \alpha+\beta+\gamma=1, \\
& \alpha, \beta \text { and } \gamma \in[0 ; 1] .
\end{aligned}
$$

4.2. AHP Method. AHP is a well known multicriteria method exploited for resolving decision problems. It has been applied in several research fields including marketing and engineering. AHP method is composed of five steps: [29].

Step 1: Determine the problem criteria.

Step 2: generate the pair wise-comparison for all criteria using the values of Table 1.

Step 3: determine the most appropriate eigenvectors to the maximal eigenvalue to define the criteria importance. 
Step 4: represent a validation step where judgments consistency is compared to Consistency Ratio (CR) and Consistency Index (CI) [30]:

$$
\mathrm{CI}=\frac{\mu_{\max }-n}{n-1},
$$

where $\mu_{\max }$ represents the Eigenvalue that corresponds to the pair-wise comparisons matrix, and $n$ represents the number of different elements considered for the comparison. CR is defined using equation:

$$
\mathrm{CR}=\frac{\mathrm{CI}}{\mathrm{RCI}}
$$

where RCI is a random consistency index chosen from Table 2 according to the number of criteria. The last step aims to validate the process using CR: pairwise comparisons are considered valid if $\mathrm{CR}$ is less or equal to 0.1 .

\subsection{Optimal User Association}

4.3.1. Our Algorithm. The steps the optimal association of users are described in Algorithm 1.

4.3.2. Numerical Illustration. The cost matrix has been applied to associate users to the most suitable BS. We use the AHP method to determine criteria weights. Our decision problem is prioritizing the available BSs.

Our decision matrix is illustrated in Table 3, where we define all criteria importance. We observe that all matrices are consistent because each consistency rate is less than 0.1 .

The normalized matrix is given in Table 4:

The next step focuses on generating criteria weights. After calculating such weights, we found that $\mathrm{C} 1=0.77$ and $\mathrm{C} 2=0.16$ and $\mathrm{C} 3=0.07$. To prove that the weights generating is valid, we calculate the CR value. Firstly, the weighted matrix is illustrated in Table 5:

LamdaMAx $=3.07 \mathrm{CI}=0.03 \mathrm{CR}=0.06$ After calculating all criteria weights using the AHP model, we use these generated weights for alternatives costs.

\section{Simulation Results}

In this section, we present the performances of the proposed joint user association. In the simulation, we examine a HetNet scenario containing multiple overlapping area access networks. We supposed that all the MUE is arbitrarily and randomly located in the area. The main parameters used in the simulation are summarized in Table 6.

5.1. Energy Efficiency and SINR. We evaluate the proposed association approach in terms of energy efficiency and SINR factors. In the studied scenario, we consider two small BS and one macro BS, while the number of mobile users is varied between 30 and 90 (Figures 2-4). (Figures 5-7), and (Figures 8-10) depict the curves of the energy efficiency and the SINR for the association of 30, 60, and 90 MUE respectively. It is clearly shown from curves that the proposed approach increased significantly the energy efficiency due to the optimal association of users. Besides, we model the energy efficiency problem as minimal cost function where the weights are calculated using the AHP method. The calculation of the weights of importance using the AHP technique has eased making the optimal user association. Moreover, we observe that the values of the SINR are decreased for the users associated with the available BS. Also, We can conclude that the user association is individual. Indeed, user15, user16, ..., and user25 (Figure 2) are not associated with macro BS because of their increased cost values. However, these users are associated with the small BSs due to their minimal cost compared to the macro BS. Hence, the interference problem is resolved.

\section{Conclusion}

In this paper, we investigated an energy-efficient user association and interference mitigation in HetNet. For user association, we simultaneously considered the SINR, power consumption, and user distance. In our algorithm, users associated with BSs depending on their cost values. This optimized energy efficiency and mitigation of interference though concurrently preventing recurrent switching between users and SBSs. The simulation results presented that our proposed strategy can optimize network performance in HetNet. As future work, we intend to optimize energy consumption in both uplink and downlink transmissions.

\section{Data Availability}

The data used to support the findings of this study are available from the corresponding author upon request.

\section{Conflicts of Interest}

The authors declare that they have no conflicts of interest.

\section{References}

[1] S. Achki, F. Gharnati, and A. Ouahman, "Enhancing energy consumption in wireless communication systems using weighted sum approach," Indian Journal of Science and Technology, vol. 10, no. 4, 2017.

[2] A. Samira, G. Fatima, and A. O. Abdellah, "Assessment of energy efficiency of base station using SMART approach in wireless communication systems: special issue on data and security engineering," in Innovations in Smart Cities Applications Edition 2, pp. 850-857, Springer, Berlin, Germany, 2019.

[3] M. Hawasli and S. Aldirmaz, "Toward green 5G heterogeneous small-cell networks: power optimization using load balancing technique," AEU-International Journal of Electronics and Communications, vol. 82, pp. 474-485, 2017.

[4] K. M. Saidul Huq, S. Mumtaz, J. Bachmatiuk, J. Rodriguez, X. Wang, and R. L. Aguiar, "Green hetnet CoMP: energy efficiency analysis and optimization," IEEE Transactions on Vehicular Technology, vol. 64, no. 10, pp. 4670-4683, 2015.

[5] A. Samira, J. Yassine, G. Fatima, and A. Abdellah, "Adaptive beamforming with MVDR algorithm for assessment energy efficiency in cellular network," International Journal of Civil 
Engineering and Technology (IJCIET), vol. 11, pp. 211-219, 2020.

[6] B. Boyd, S. Talwar, R. Arefi, and K. Stewart, "Networks and devices for the 5G era," IEEE Communications Magazine, vol. 52, no. 2, pp. 90-96, 2014.

[7] L. Tang, H. Hu, and Y. He, "Energy efficient joint power control and user association optimization in massive mimo enabled hetnets," Applied Sciences (Switzerland), vol. 8, no. 4, p. 584, 2018.

[8] C. Weeraddana, M. Codreanu, M. Latva-aho, A. Ephremides, and C. Fischione, "Weighted sum-rate maximization in wireless networks: a review," Foundations and Trends in Networking, vol. 6, no. 1-2, pp. 1-163, 2011.

[9] C. Kim, R. Ford, and S. Rangan, "Joint interference and user association optimization in cellular wireless networks," in Proceedings of the 48th Asilomar Conference on Signals, Systems and Computers, ACSSC 2014, pp. 511-515, Pacific Grove, CA, USA, November 2014.

[10] P. Ameigeiras, J. Navarro-Ortiz, P. Andres-Maldonado et al., "3GPP QoS-based scheduling framework for LTE," EURASIP Journal on Wireless Communications and Networking, vol. 2016, no. 1, 12 pages, 2016.

[11] X. Ge, X. Li, H. Jin, J. Cheng, and V. C. M. Leung, "Joint user association and user scheduling for load balancing in heterogeneous networks," IEEE Transactions on Wireless Communications, vol. 17, no. 5, pp. 3211-3225, 2018.

[12] S. Deb, P. Monogioudis, J. Miernik, and J. P. Seymour, "Algorithms for enhanced inter-cell interference coordination (eICIC) in LTE hetnets," IEEE/ACM Transactions on Networking, vol. 22, no. 1, pp. 137-150, 2014.

[13] R. Chai, M. Chen, Q. Chen, and Y. Gao, "An optimal joint user association and power allocation algorithm for secrecy information transmission in heterogeneous networks," Wireless Communications and Mobile Computing, vol. 2017, Article ID 5120538, 13 pages, 2017.

[14] T. Zhou, N. Jiang, Z. Liu, and C. Li, "Joint cell activation and selection for green communications in ultra-dense heterogeneous networks," IEEE Access, vol. 6, pp. 1894-1904, 2017.

[15] H. Boostanimehr and V. K. Bhargava, "Unified and distributed QoS-driven cell association algorithms in heterogeneous networks," IEEE Transactions on Wireless Communications, vol. 14, no. 3, pp. 1650-1662, 2015.

[16] S. Patil and A. M. Bhavikatti, "Heterogeneous network optimization using robust power-and-resource based algorithm," International Journal of Electrical and Computer Engineering (IJECE), vol. 9, no. 5, p. 4226, 2019.

[17] M. Feng, S. Mao, and T. Jiang, "Base station on-off switching in 5G wireless networks: approaches and challenges," IEEE Wireless Communications, vol. 24, no. 4, pp. 46-54, 2017.

[18] A. Imran, F. Boccardi, and L. Ho, "Sleep mode techniques for small cell deployments," IEEE Communications Magazine, vol. 49 , no. 8 , pp. 72-79, 2011.

[19] C. Liu, B. Natarajan, and H. Xia, "Small cell base station sleep strategies for energy efficiency," IEEE Transactions on Vehicular Technology, vol. 65, no. 3, pp. 1652-1661, 2015.

[20] L. Qi and L. Yiqun, "Energy conservation schemes based on mobility modeling in small cell networks," in Proceedings of the 2014 XXXIth URSI General Assembly and Scientific Symposium (URSI GASS), pp. 1-4, Beijing, China, August 2014.

[21] J. Gao, Q. Ren, P. S. Gu, and X. Song, "User association and small-cell base station on/off strategies for energy efficiency of ultradense networks," Mobile Information Systems, vol. 2019, Article ID 6871378, 12 pages, 2019.
[22] A. K. Sangaiah, A. A. R. Hosseinabadi, M. B. Shareh, S. Y. B. Rad, A. Zolfagharian, and N. Chilamkurti, "IoT resource allocation and optimization based on heuristic algorithm," Sensors, vol. 20, no. 2, p. 539, 2020.

[23] A. K. Sangaiah, M. Sadeghilalimi, A. A. Rahmani Hosseinabadi, and W. Zhang, "Energy consumption in point-coverage wireless sensor networks via bat algorithm," IEEE Access, vol. 7, pp. 180258-180269, 2019.

[24] A. K. Sangaiah, D. V. Medhane, T. Han, M. S. Hossain, and G. Muhammad, "Enforcing position-based confidentiality with machine learning paradigm through mobile edge computing in real-time industrial informatics," IEEE Transactions on Industrial Informatics, vol. 15, no. 7, pp. 4189-4196, 2019.

[25] A. K. Sangaiah, D. V. Medhane, G.-B. Bian, A. Ghoneim, M. Alrashoud, and M. S. Hossain, "Energy-aware green adversary model for cyber physical security in industrial system," IEEE Transactions on Industrial Informatics, vol. 16, no. 5, pp. 3322-3329, 2020.

[26] L. Aziz and H. Aznaoui, "Efficient routing approach using a collaborative strategy," Journal of Sensors, vol. 2020, Article ID 2547061, 17 pages, 2020.

[27] Y. J. Chun, M. O. Hasna, and A. Ghrayeb, "Modeling and analysis of HetNet interference using poisson cluster processes," in Proceedings of the 2014 IEEE 25th Annual International Symposium on Personal, Indoor, and Mobile Radio Communication (PIMRC), pp. 681-686, Washington, DC, USA, September 2015.

[28] S. Song, Y. Chang, X. Wang, and D. Yang, "Coverage and energy modeling of HetNet under base station on-off model," ETRI Journal, vol. 37, no. 3, pp. 450-459, 2015.

[29] L. Aziz, S. Raghay, and H. Aznaoui, "An improved multipath routing protocol using an efficient multicriteria sorting method: special issue on data and security engineering," in Innovations in Smart Cities Applications Edition 2, pp. 837-849, Springer, Berlin, Germany, 2019.

[30] J.-T. Hsueh and C.-Y. Lin, "Integrating the AHP and topsis decision processes for evaluating the optimal collection strategy in reverse logistic for the TPI," International Journal of Green Energy, vol. 14, no. 14, pp. 1209-1220, 2017. 